\title{
VIOLENCIA ESTRUCTURAL, CRIMMENES CONTRA LA HUMANIDAD, Y GARANTIAS DE NO REPETICIÓN EN EL CONTEXTO COLOMBIANO
}

\section{STRUCTURAL VIOLENCE, CRIMES AGAINST HUMANITY, AND GUARANTEES OF NON-REPETITION IN COLOMBIAN CONTEXT}

\author{
Karina Johana Martínez Jiménez \\ Universidad de Cartagena, Cartagena de Indias, Colombia \\ kmartinezj25@gmail.com
}

Palabras clave: Teoría política, teoría social, crímenes contra la humanidad, derechos humanos, pobreza, Colombia, reforma rural.

Keywords: Political theory, social theory, crimes against humanity, human rights, poverty, Colombia, rural reform.

Resumen: El Acuerdo de paz firmado en 2016 entre el gobierno de Juan Manuel Santos y las Fuerzas Armadas Revolucionarias de Colombia (FARC) contiene un enfoque integral en cuanto a la no repetición de la violencia en el marco del conflicto. Donde uno de los puntos consignados es la Reforma Rural Integral (punto 1). El objetivo general del presente trabajo es realizar una aproximación a la proyección de esta última como una de las garantías de no repetición, mediante un estudio de la violencia estructural y los crímenes contra la humanidad en el país. Lo anterior se desarrolla mediante una metodología descriptiva y crítica del fenómeno de la violencia, pero también desde un enfoque sociológico, en cuanto este último persigue la comprensión de las relaciones sociales objetivas e históricas del fenómeno. De la lectura realizada se puede concluir que el punto 1 aporta una contribución significativa para la no repetición de los crímenes contra la humanidad, ya que plantea mecanismos para la disminución de la violencia estructural, en materia de derechos humanos y derechos socioeconómicos.

Abstract: The peace agreement signed in 2016 between the government of Juan Manuel Santos and the Fuerzas Armadas Revolucionarias de Colombia (FARC) contains a comprehensive approach to the non-repetition of violence in the context of the conflict. One of the agreed points is comprehensive rural reform (Item point 1). The general object of this work is to approach to the projection of the latter as one of the guarantees of non-repetition, based on 
a study on structural violence and crimes against humanity in the country. The foregoing is developed through a descriptive and critical methodology of the phenomenon of violence, but also from a sociological analysis, insofar as the latter seeks to understand the objectiveand historical social relations of the phenomenon. From the reading carried out, it can be concluded that Item 1 makes a significant contribution to the nonrepetition of crimes against humanity, since it raises mechanisms to reduce structural violence, in terms of human rights and socio-economic rights.

\section{Introducción}

La violencia sistemática que ha azotado a Colombia desde el siglo pasado plantea un llamado más allá de una solución de carácter militar. Si bien es cierto que muchas zonas del país necesitan la presencia del Estado, esta no debe reducirse a un aumento en la militarización, sino que es necesario intervenir en materia de políticas públicas eficaces para garantizar los derechos fundamentales de la población civil. El informe del Alto Comisionado de las Naciones Unidas para los Derechos Humanos (2020), ha manifestado que en aquellas zonas objeto de intervención integral por parte del gobierno nacional, ha primado la presencia de la fuerza pública. Escenario en el cual la comisión de crímenes contra la humanidad no parece llegar a su fin. Más allá de un endurecimiento en la militarización de las zonas más afectadas, se requiere una atención a las causas estructurales de la violencia, de una presencia institucional tanto en ámbitos de justicia como de garantía de derechos sociales y económicos.
De la misma manera, el desarrollo de la política para el desmantelamiento de las organizaciones criminales, así como la solución a la cuestión de los cultivos ilícitos.

En 2016, y luego de un proceso de negociación entre el gobierno de Juan Manuel Santos (2010-2018) y la guerrilla de las Fuerzas Armadas Revolucionarias de Colombia (FARC), se pactó un acuerdo cuyo fin no solo pretendía la desmovilización de los insurgentes, sino que impulsaría la construcción de las condiciones para alcanzar "una paz estable y duradera". El Acuerdo, además de ser un instrumento para la transición, representa una contribución para el mejoramiento de las circunstancias estructurales sobre las que ha tenido lugar el círculo de la violencia. Aquí, las garantías de no repetición trascienden el dilema entre justicia retributiva y restaurativa, pues al tiempo que se utilizan estos mecanismos de justicia, se requiere establecer otros que contribuyan a la disminución de los factores estructurales de la violencia.

En dicho acuerdo se ha establecido que las garantías de no repetición requieren de la implementación integral de todos los puntos acordados (Acuerdo final, 2016). Estos tratan desde la cuestión sobre los cultivos de uso ilícito, la política para el desmantelamiento de las organizaciones criminales, el desarme y desmovilización de los insurgentes, la participación política y ciudadana, el sistema de justicia y derechos de las víctimas, hasta la necesaria transformación del campo mediante una reforma rural integral. El objetivo del presente trabajo es reflexionar acerca de la proyección de esto último, mediante un análisis descriptivo del contexto de la violencia en Colombia y las condiciones estructurales que caracterizan a la población más afectada por las dinámicas de la 
guerra y la comisión de crímenes contra la humanidad. Desde una perspectiva sociológica, se busca atender a la comprensión de la violencia mediante la descripción de las relaciones de exclusión social como fenómeno transversal en el desarrollo de los escenarios violentos.

Dicho de otro modo, para una aproximación hacia la contribución de la Reforma Rural Integral (punto 1) a la no repetición, es necesario atender al análisis de la violencia estructural y las dinámicas de los conflictos, así como hay que tener en cuenta quién es la población mayormente afectada en el territorio nacional. Desde el punto de vista sociológico, que intenta explicar un fenómeno social mediante las pautas y las formas en que este se organiza (Fariñas, 1998), resulta relevante un análisis descriptivo de la violencia, por cuanto este permite comprender en un sentido objetivo, o material de las relaciones sociales, el contexto y circunstancias estructurales del fenómeno en cuestión.

De acuerdo con el análisis, se puede afirmar que en el caso colombiano como en la teoría galtungiana, a mayor violencia estructural, mayor probabilidad de violencia directa (Hueso, 2000). De manera que la reforma rural representa una contribución significativa ya que aporta mecanismos sociales y políticos que contribuyen a la transformación de un estatus de vulnerabilidad. Esto último es de gran relevancia por cuanto existen altos niveles de precarización social y detrimento de las garantías de derechos humanos para la población rural más victimizada.

Para el desarrollo del trabajo, en una primera parte se realiza un análisis descriptivo del contexto de la violencia, así como la relación entre la violencia estructural y la violencia directa en el país (epígrafe 2).
En una segunda parte, se describe la situación de los crímenes contra la humanidad hacia los líderes sociales y defensores de derechos humanos en una etapa de post-acuerdo (epígrafe 3). En la tercera parte se realiza un esbozo del punto 1 del Acuerdo, no solo por considerarse uno de los puntos centrales, sino por su contribución al mejoramiento de uno de los factores de la violencia estructural en territorio colombiano. Esto es, lo que tiene que ver con las condiciones del campo y la comunidad rural más afectada por los altos niveles de pobreza, de exclusión social o de vulneración de derechos fundamentales (epígrafe 4). Finalmente, se exponen algunas conclusiones (epígrafe 5).

\section{Contexto de la violencia en Colombia. Dinámicas complejas}

El conflicto interno colombiano que en los años 60s aparecía como un enfrentamiento entre el Estado y las guerrillas se ha complejizado y se ha sumido en altos niveles de degradación. Desde los años 80s con la incursión en el narcotráfico por parte de grupos paramilitares ha aumentado significativamente la intensidad de la violencia. Aunque en un inicio el objetivo de estos grupos se había dirigido a la lucha contrainsurgente, se han enfocado mayormente al control territorial de los cultivos de uso ilícito o las rutas para su transporte, pero también por la minería y otros recursos naturales. Por su parte, los fines políticos de las guerrillas de izquierda se tornaron borrosos al recurrir a prácticas igualmente degradantes, verbigracia, financiación de economías ilegales, o el secuestro (Echandía, 2008). 
De modo que las dinámicas de la violencia en el contexto colombiano se han relacionado en sus orígenes con la lucha guerrillera por la transformación política, hasta continuar en las complejas luchas por el poder y control de las economías ilegales por parte de los actores armados involucrados. En este contexto, la población civil ha sufrido los mayores niveles de victimización en las distintas modalidades -tanto por desplazamiento forzado, violencia sexual, asesinato y otras modalidades de violencia-. Mientras que los victimarios conforman un complejo de actores entre guerrillas, paramilitares, bandas criminales del narcotráfico, organizaciones transnacionales, pero también instituciones militares del Estado, quienes han participado en la comisión de ejecuciones extrajudiciales y que históricamente han colaborado con organizaciones paramilitares en la lucha contrainsurgente (Gómez Isa, 2008).

Además de la explicación del conflicto por cuanto el control estratégico de las zonas, las formas en que este organiza y continúa en el tiempo son múltiples y complejas. Estos van desde control y disputas políticas, económicas, sociales, históricas, o fenómenos como la corrupción institucional. No obstante, entre los elementos objetivos $^{1}$ e históricos que han sido reconocidos como precursores del conflicto y sus efectos se encuentra la persistencia de altos niveles de exclusión social, po-

1. El fenómeno de la violencia puede ser estudiado a partir de factores subjetivos u objetivos. Los primeros argumentan el uso de la violencia por los deseos de la adquisición de poder o bienes, por parte de seres racionales; mientras que los segundos atienden a las condiciones sociales como la exclusión o los altos niveles de precarización de la vida, y la facultad institucional para responder a estos fenómenos (Valencia y Cuartas, 2009). breza o falta de derechos fundamentales en los territorios (Bonett, 2001; Hernández, 2012; Salas, 2016). Este estado de cosas ha incentivado la creación y reorganización de grupos por el control territorial de las economías ilícitas. Asimismo, la precarización social se ha convertido en un terreno abonado para la captación del recurso humano de organizaciones al margen de la ley (Bonett, 2001).

Aunque la precarización social no es el único factor -entre otros se encuentra el auge de la economía ilícita, la colonización alrededor de las zonas de prosperidad de explotación minera, o la corrupción política (Pécaut, 1997, Bonett, 2001)-, representa uno de los elementos que ha contribuido con el ciclo de la violencia. Es así que las zonas más afectadas por la incidencia criminal de grupos armados al margen de la ley son las llamadas "zonas de alto riesgo", que al mismo tiempo presentan niveles significativos de precarización de derechos sociales (Bonett, 2001; Hernández, 2012; Salas, 2016; ACNUDH, 2019, 2020). Recurrir a estos datos históricos permite entonces entender la relación en paralelo y de sustento entre la violencia estructural (condiciones materiales de las relaciones sociales como exclusión, control de poder) y la violencia directa (atentado en contra de la vida u otros bienes jurídicos de la población civil).

\section{I Violencia estructural y violencia directa}

Existe un consenso en la idea de que la violencia no es una cualidad innata de los seres humanos -como sí lo es el conflicto-, sino que es una característica cultural aprendida a lo largo del ciclo de 
la vida. En esta línea, la violencia entendida como una "evolución condicionada por el entorno social" (Jiménez-Bautista, 2012: 17), permite inferir que para minimizar sus efectos, es necesario atender a sus causas sociales. Desde una visión antropológica, la guerra no es producto de instintos, sino más bien de las condiciones y necesidades materiales y culturales que se producen en todas las sociedades. En la línea de investigadores como Johan Galtung, "una definición genérica de la violencia podría corresponderse a todo aquello que, siendo evitable, impide, obstaculiza o no facilita el desarrollo humano o el crecimiento de las capacidades potenciales de cualquier ser humano" (Jiménez-Bautista, 2012: 18).

Desde la teoría de Johan Galtung se entiende la "violencia directa" como los efectos visibles de esta, verbigracia las muertes, los heridos o desplazados. Mientras que la violencia estructural es aquella originada por la injusticia y la desigualdad desde la estructura social. También se habla de un tercer tipo de violencia, llamada cultural, la cual legitima y promueve las relaciones estructurales hegemónicas. De manera que para la consecución de la paz se necesita trabajar en las tres al mismo tiempo (Hueso, 2000). Donde la violencia estructural tiene una relación proporcional con la violencia directa (Calderón, 2009).

De manera que "si no se hace nada por atajar el conflicto en las raíces del mismo, tarde o temprano la violencia volverá a surgir cuando los horrores del último estallido hayan desaparecido de la memoria colectiva" (Hueso, 2000). Frenar la violencia directa implica entonces darle tratamiento inmediato a la violencia estructural y cultural evitando la circularidad de la violencia. Pero también hay que tener en cuenta que no es común un conflicto con dos únicos actores u objetivos, y esto no solo por la multiplicidad de intereses, sino porque estos mismos pueden evolucionar con el paso del tiempo (Hueso, 2000).

Si bien no se puede reducir como única causa de los conflictos el factor material o socioeconómico, este sí representa uno de los elementos fundamentales para entender y atender las causas del desarrollo y continuidad de la violencia en el contexto colombiano. No se puede olvidar que este último tiene dinámicas complejas y sujetas a la multiplicidad de los actores involucrados, así como a la variedad y evolución de los intereses implicados. Ya ha sido reconocida la relación entre los índices de violencia directa y la precarización social del territorio nacional (Hernández, 2012; Bonett, 2001; Egea y Soledad, 2007; Salas, 2016; ACNUDH, 2019, 2020), que por lo general se presenta en paralelo o en proporcionalidad. Con lo cual, atender a este factor es indispensable para la construcción de una paz a largo plazo.

Aunque las dinámicas del conflicto armado se han degradado por el accionar de economías ilegales u otro tipo de actividad ilícita para efectos de financiamiento o mero lucro, el problema estructural siempre ha jugado un papel preponderante en el surgimiento de la violencia (Bonett, 2001). Es conocida la relación entre la presencia de grupos ilegales y el ejercicio de la violencia en los territorios ${ }^{2}$ (Salas,

2. Colombia no solo cuenta con una historia de violencia política, también, y sobre todo desde los 70s han incursionado intereses ligados a fenómenos como la economía ilegal. Aquí los límites entre los intereses de unos y otros actores armados muchas veces aparecen borrosos (Pécaut, 1997). 
2016). Donde hay que tener en cuenta que violencia directa que victimiza mayormente a la población civil no es el producto de dos únicos actores en conflicto, ya que el contexto colombiano abarca una complejidad de organizaciones y de intereses entre los distintos involucrados.

Pero, además, ante todos estos factores, protagonistas, e intereses encontrados se puede establecer un elemento transversal, esto es, la precariedad institucional para atender los territorios afectados (Pécaut, 1997). Aunque este último elemento suele presentarse como uno más en la cadena que sostiene el círculo de violencia sistemática, parece ser el que ha atravesado todas las dinámicas de la violencia. La ausencia institucional o su déficit tiene distintas manifestaciones que van desde lo judicial, económico, militar, social, y por supuesto, como garante de derechos básicos y políticas públicas efectivas ${ }^{3}$. Según autores como Pécaut (1997: 12), "son el quebrantamiento de las regulaciones institucionales y la pérdida de credibilidad del orden legal los que abren el campo a la violencia generalizada". De donde se deriva que las zonas más afectadas por la falta de las instituciones, sean más susceptibles a la presencia y control por parte de organizaciones criminales.

La ineficacia del gobierno ante las demandas populares ya sea por corrupción o por falta de políticas públicas eficaces ha ido en detrimento de los derechos fundamentales de la población (Egea y Soledad, 2007; Duque y Torres, 2015). Este déficit de derechos humanos en distintos

3. Históricamente, las capas más desprotegidas de la sociedad colombiana han sido las más afectadas por las dinámicas de la violencia (Pécaut, 1997). niveles, permite contrastar con las tres dimensiones galtungianas de la violencia. En este contexto el aspecto estructural se observa como uno de los factores significativos dentro de las dinámicas de los conflictos y su continuidad.

Uno de los elementos de la violencia estructural en el caso colombiano ha sido la cuestión de la pobreza y desigualdad sobre todo en las zonas rurales. Esto último tiene que ver por un lado, con la precariedad en el acceso a servicios públicos esenciales como el agua, la energía, la salud o la educación. Y por otro, con el déficit de acceso a la tierra (Alviar, 2010). Los altos niveles de concentración de la tierra y desaprovechamiento de esta han persistido, de la misma forma que sobrevive la carencia de servicios esenciales y derechos fundamentales en amplias zonas del país (Alviar, 2010; Matias, 2017).

En efecto, el territorio nacional se ha caracterizado por la exclusión de los sectores sociales más humildes (Capera y Ñañez, 2017). Y las ambigüedades en las políticas agrarias, en los objetivos legales, y económicos, han determinado en gran medida la ineficacia de las instituciones en la práctica (Alviar, 2010). A estos fenómenos estructurales se suma la presencia de organizaciones criminales, la cuestión sobre el narcotráfico, la falta de políticas públicas eficaces y garantistas de los derechos humanos (ACNUDH, 2020). Una cadena de factores a los que se une el fenómeno de la corrupción y una "práctica política defectuosa" (Bonett, 2001). En suma, un complejo de dinámicas sostiene las prácticas de violencia, y propicia un terreno más dispuesto para la criminalidad, que se enfoca en aquellos sectores más empobrecidos y con falta de institucionalidad en todos los niveles. 


\subsection{Aqudización de la victimización: ¿Una cuestión geoestratégica y socioeconómica?}

En Colombia, la violencia sistemática por lo general afecta más fuertemente aquellos territorios con presencia de organizaciones criminales y precariedad de derechos socio-económicos (ACNUDH, 2019, 2020). Para los grupos armados al margen de la ley, una de las zonas con presencia estratégica ha sido la región suroccidental, sobre todo departamentos como Nariño, Cauca, Valle, sur del Chocó, occidente del Putumayo y zona cafetera. También la región noroccidental en Arauca, Casanare y los santanderes (Bonett, 2001). Estos cuentan con un gran potencial económico y de comunicación estratégica. Pero esta situación se ha visto agravada por los altos índices de desempleo, narcotráfico, corrupción y delincuencia común. El desempleo, Ios bajos niveles de educación, así como el hambre, y otros fenómenos socioeconómicos, además de dejar en alto grado de vulnerabilidad a la población, predisponen el recurso humano para la criminalidad (Bonet, 2001).

Otras zonas con mayor presencia de grupos armados han sido San Vicente del Caguán, parte del Meta, Caquetá, y sur de Bolívar. Este último con una porción considerable del rio Magdalena, y la Serranía de San Lucas. Las zonas representan puntos estratégicos para el cultivo y el transporte de ilícitos, así como fuentes de recursos naturales ligados a industrias como la minería, o el petróleo. Asimismo, la mayoría de estos territorios se caracterizan por la poca presencia institucional y su lejanía de los principales centros económicos y de toma de decisiones políticas (Bonett, 2001; Egea y Soledad, 2007; Matias, 2017).

También es cierto que existe un histórico desequilibrio social entre las comunidades rurales y urbanas, un abandono institucional en vastos sectores rurales (García, 2019). Donde los territorios campesinos y sus sectores sociales más excluidos han recibido el mayor impacto de la violación de derechos humanos. Cobra importante relevancia que la mayor tensión entre el Estado y la sociedad civil se haya agudizado en zonas donde ha existido mayor precariedad de las condiciones socio-económicas (Capera, y Ñañez, 2017). Es así que la población más afectada por los efectos del conflicto o el recrudecimiento de la violencia han sido sectores rurales, comunidades ancestrales 0 , en fin, sectores más pobres de la población civil:

(...) muchas veces, quienes han recibido su mayor impacto han sido generalmente las poblaciones más vulnerables de este país, a cuyos territorios llegó la confrontación armada sin haberlo pedido, sin que hubiesen hecho una opción por esta modalidad de violencia, aunque a pesar de ello, comprometen valiosos esfuerzos para sobrevivir, proteger derechos fundamentales, proponer y transformar desde métodos no violentos (Hernández, 2012: 41).

Entre las comunidades más afectadas se encuentran las raizales, quienes han sufrido de fenómenos como la "desterritorialización" en regiones como el pacífico. Ello debido a la acción de actores armados, pero que también se ha relacionado con intereses económicos de otro tipo que van desde megaproyectos, carreteras, hasta el narcotráfico en los procesos de apropiación de la región (Oslender, 2004). La región pacífica, con más del 80 
\% de población afrocolombiana, seguido de grupos indígenas, se ha reconocido como una de las más olvidadas y pobres del país. No obstante, históricamente ha sido objeto de proyectos de desarrollo como las plantaciones de palma africana, o las camaroneras (Escobar, 2004). Situación que pone en evidencia el interés por un beneficio externo, por encima del bienestar social de las comunidades.

Por su parte, la gestión militarista de la violencia, como la política de seguridad democrática en 2002, ha demostrado que, si no se realiza una intervención integral, se agudizan los niveles de pobreza y exclusión social (Bello, 2003). Aunque la violencia directa se lleva a cabo según cierta configuración territorial en función de variables como el cultivo de ilícitos, la movilidad, o la extracción de recursos naturales (Salas, 2016), factores como el desempleo, y aquellos relacionados con la falta de garantías sociales y déficit institucional, aumentan las probabilidades de victimización en dichas zonas (Bonett, 2001).

En efecto, la precarización social como elemento de la violencia estructural, ha encontrado una relación en paralelo con el recrudecimiento de la violencia directa, convirtiéndose en contribuyente relevante para esta última. Situación de exclusión que ha creado las condiciones para que el conflicto y la violencia sistemática tenga tierra fértil para su desenvolvimiento (Jaramillo, 2015). Véase, en lo que sigue, cómo se articula la precarización social en los territorios, con la violencia sistemática, o más exactamente, con la comisión de crímenes contra la humanidad.

\section{Crímenes contra la humanidad en el caso de líderes sociales y defensores de derechos humanos en el post-acuerdo}

Desde inicios del siglo XXI, la situación de los defensores de derechos humanos se convirtió en un tema relevante para organismos internacionales como Naciones Unidas, que la llevó a crear la figura del Relator Especial sobre la situación de las y los defensores de derechos humanos $^{4}$. En Colombia, luego de la firma del Acuerdo de paz de 2016, la comisión de delitos internacionales en contra de esta población continúa siendo una preocupación vigente. De hecho, lo que se ha visto es un recrudecimiento de la violencia en contra de este grupo social, y la falta de una postura "unánime" por parte del gobierno acerca de esta situación (Ball, Rodríguez, y Rozo, 2018).

La Oficina del Alto Comisionado de las Naciones Unidas para los Derechos Humanos en Colombia ${ }^{5}$ ha resaltado que las comunidades más afectadas por este tipo de victimizaciones se caracterizan por la persistencia de deficiencias en derechos socioeconómicos, culturales, o de acceso a la justicia: "estas causas generan altos índices de pobreza multidimensional

4. Véase, https://www.ohchr.org/sp/Issues/ SRHRDefenders/Pages/SRHRDefendersIndex. aspx (07/09/19).

5. Informe $\mathrm{A} / \mathrm{HRC} / 40 / 3 / \mathrm{Add} .3$ accesible en https://www.hchr.org.co/index.php/informes-ydocumentos/informes-anuales/9017-informedel-alto-comisionado-de-las-naciones-unidaspara-los-derechos-humanos-sobre-la-situacionde-derechos-humanos-en-colombia-durante-elano-2018 (28/08/19). 
y propician el surgimiento de economías ilícitas, controladas o disputadas por grupos criminales, lo que también provoca niveles endémicos de violencia" (OACNUDH, 2019: 4). Para continuar con los objetivos del trabajo, es necesario exponer qué se entiende por crímenes contra la humanidad, ya que estos continúan afectando la situación de derechos humanos de los líderes sociales en el post-acuerdo, y las garantías de no repetición deben dirigir los esfuerzos según el contexto en el que ocurren.

\section{3.l Qué es el crimen contra la humanidad}

A partir del Estatuto de Roma de 1998, se entiende como crímenes contra la humanidad aquellos delitos con carácter generalizado, sistemático y con conocimiento del ataque en contra de una población civil (Ambos, 2013). Estos se dan en un contexto de impunidad o tolerancia institucional (Bolívar, 2011). El carácter sistemático tiene que ver con la existencia de un plan o política criminal, mientras que lo generalizado se refiere a una cantidad masiva de casos (Forer y López, 2010). Cuando se habla de este tipo de crimen se entiende que el perpetrador cuenta con medios que no tiene el autor de un delito común, de donde deriva la repetición de los casos y la impunidad para los victimarios. Estos últimos, sean organismos estatales o no, son aquellos que cuentan con estructuras criminales, control económico, político, y social en Ios territorios (Ambos, 2013; Gil, 2016; Liñán, 2017). En cuanto a las víctimas, se caracterizan por ser miembros de la población civil y por lo general relacionados con un grupo social, sea étnico, político, religioso, entre otros.
Se entiende que la comisión de estos crímenes es posible en un contexto de impunidad. Esta puede ser entendida en dos sentidos, aunque relacionados en la práctica: impunidad de facto e impunidad normativa. La primera se refiere a situaciones donde, pese a la existencia de normativa, las situaciones de hecho impiden la persecución de los responsables. La mayoría de las veces debido al control territorial de grupos criminales. Mientras que la segunda tiene que ver con la imposibilidad jurídica de iniciar la persecución penal que corresponde (Gil, 2003; Chinchón, 2012). De manera que la ejecución de este tipo de crímenes encuentra mayor probabilidad para su ejecución en aquellas zonas controladas por organizaciones criminales, debido en muchos casos, a la ineficaz acción de las instituciones y las condiciones de vulnerabilidad de la población.

\subsection{Crímenes contra la humanidad en el caso de líderes sociales}

Según el informe ¿Cuáles son los patrones? Asesinatos de líderes sociales en el post acuerdo, se han cometido 257 casos de asesinatos sistemáticos entre el 24 de noviembre de 2016 hasta el 31 de julio de 2018. Estos delitos han sido perpetrados mayormente en zonas rurales. El 70, $42 \%$ se concentró en 8 departamentos de los 32 del país: Cauca, Antioquia, Norte de Santander, Valle del Cauca, Córdoba, Putumayo, Nariño y Chocó (CINEP, 2018: 216). Dichas regiones se han caracterizado por una alta presencia de grupos armados, altos niveles de marginalización, escasa presencia institucional civil, persistente violencia estructural, rutas del narcotráfico, actividades extractivas. 
Pero también zonas donde se lleva a cabo la implementación de mecanismos del Acuerdo de paz de 2016, dirigidos a programas de desarrollo o espacios territoriales de capacitación y reincorporación ETCR (CINEP, 2018: 217). La persistencia de estructuras criminales a cargo de las economías ilícitas y las rentas que esta genera, parecen continuar la disputa en los territorios, y ello aún más cuando los planes de desarrollo social no se implementan oportunamente y existe un recurso humano disponible.

En cuanto a las víctimas, estas se caracterizan por el arraigo local y el liderazgo en sus comunidades. El asesinato de líderes indígenas representa el 12,06 \%, y los líderes afrodescendientes el 9,33 \% del total. Por lo que estas violaciones afectan con mucha fuerza a grupos étnicos que en su mayoría viven en condiciones de precariedad. Entre las actividades de liderazgo desarrolladas se encuentran precursores de restitución de tierras, movimientos políticos, defensores de derechos de las víctimas, defensores del Acuerdo de paz, hasta activistas defensores de derechos humanos (CINEP, 2018).

Si bien las víctimas dentro de esta categoría no necesariamente pertenecen a un grupo social homogéneo -hay campesinos, líderes comunales, étnicos, víctimas, hasta defensores del Acuerdo de paz-, son potencialmente objeto de identificación por parte de las organizaciones criminales o grupos económicos. Lo anterior por cuanto que representan una población que interfiere los intereses de poder y control que los victimarios tienen sobre los territorios. Aquí, la selectividad de este grupo poblacional es posible por el ejercicio activo que desempeñan, así como por la presencia de las estructuras criminales que ejercen el control social y económico (CINEP, 2018).
Por su parte, el carácter generalizado de estos crímenes no solo se ve reflejado por el número de víctimas. Donde las cifras no dejan de generar debate por cuanto distintas organizaciones tienen estimaciones diferentes, e incluso trabajos como los de Ball, Rodríguez y Rozo (2018) han estimado un subconteo de los asesinatos en los años 2016 y 2017. Más aún, dicha generalización puede verse también por la continuidad en el tiempo de estos delitos o de la llamada impunidad de facto. Esto ya sea por la persistencia del control territorial de los grupos criminales, o por la falta de justicia efectiva. Respecto a esto último, pese a que se ha observado un significativo avance procesal en las investigaciones de aproximadamente el $27 \%$ de los casos, se afirma que aproximadamente el 90\% de estos entre 2009 y 2017 continuaban en la impunidad (ClNEP 2018: 167).

Existe entonces una identificación de la población victimizada, así como la impunidad en el tiempo y el control territorial por parte de estructuras criminales. Pero además, como se verá a continuación, la población mayormente afectada por este tipo de violencia pertenece a territorios con altos niveles de precarización social y abandono institucional, donde dichas estructuras criminales encuentran mejores circunstancias para el ejercicio de poder y represión. Este aspecto es importante para entender el porqué de las medidas a propósito de los acuerdos para la transición deben atender no solo al castigo de los responsables de la comisión de los crímenes contra la humanidad, sino que al mismo tiempo los mecanismos que se adopten deben tener en cuenta el contexto, la realidad política y social de la mayoría de víctimas. Ello para que, a la par de una perspectiva de "reparación 
transformadora" (Uprimny, 2009; 2016), se persiga el cambio y se desarrolle una política social que atienda al mejoramiento de las condiciones de vulnerabilidad de las comunidades.

\subsection{Territorialización de la violencia sistemática}

Históricamente, la gestión del conflicto por parte de las instituciones se ha enfocado mayormente en un punitivismo ${ }^{6}$ que concentra los esfuerzos del sistema y hace un énfasis en el castigo. Ha prevalecido un discurso que enfatiza en el derecho penal como mejor herramienta para combatir la violencia, pese a que más de la mitad de los casos conocidos por las autoridades parecen quedarse sin resolver ${ }^{7}$. De manera que la inversión en materia de justicia se ha concentrado mayormente en el ámbito de lo penal (Ardila, 2018: 182). Al mismo tiempo, la militarización se ha superpuesto en la intervención institucional (ACNUDH, 2020), y han continuado las denuncias por parte de la sociedad civil en cuanto al ejercicio de la violencia de la fuerza pública, por ejemplo, en relación a la política de erradicación forzada de cultivos de uso ilícito (Indepaz, 2020).

Pero lo cierto es que la agudización de la violencia sistemática ha persistido en los

6. El punitivismo puede ser entendido como un apoyo o énfasis en el castigo penal rigorista, o un endurecimiento punitivo (Cerezo, 2010) y, en últimas, en la idea de que la severidad de la pena es la mejor forma de combatir la delincuencia (Cancio, 2010; Gil, 2014). En la línea de Landa (2018), cuando dicho punitivismo es excesivo, tiende a convertir el derecho penal en "un instrumento de venganza" (Landa, 2018: 4).

7. Al respecto véase, Ardila, 2018, pp.152, nota 159. territorios más vulnerables y las llamadas "zonas de alto riesgo" (Antioquia, Córdoba, Norte de Santander, Arauca, Chocó, Cauca, Valle del Cauca, Putumayo y Nariño). De ello se deriva que de la mano de la militarización y el mejoramiento del sistema penal, se debe reforzar la institucionalidad en materia de garantías de derechos humanos (ACNUDH, 2020). Como lo ha demostrado la persistente comisión de crímenes contra la humanidad, el efecto disuasorio que representa la pena o la incursión de militares a las zonas, no ha tenido el impacto suficiente para frenar la violencia. Ante este escenario, se requiere atender a los factores que en materia social intervienen en la violencia estructural $^{8}$.

Según el informe anual del Alto Comisionado de las Naciones Unidas para los Derechos Humanos (ACNUDH), los asesinatos de defensores de derechos humanos en el 2019 tuvieron un impacto del $86 \%$ en aquellos municipios con altos índices de pobreza multidimensional ${ }^{9}$,

8. El informe anual del ACNUDH ha expuesto que pese al decreto 2278 de 2019 del gobierno nacional que prioriza la presencia estatal en las "zonas estratégicas de intervención integral", se ha observado una presencia más militarizada que en materia de garantía de derechos e inversión social (ACNUDH, 2020). Decreto 2278 de 2019 accesible en https://www.funcionpublica.gov.co/ eva/gestornormativo/norma.php?i=104212. Por su parte, como pudo verse luego de la desmovilización de paramilitares mediante la ley 975 de 2005 , muchos de los desmovilizados reconocieron que volver a delinquir era una posibilidad dada las condiciones de pobreza y falta de medios para sobrevivir (Duque y Torres, 2015).

9. Según el Programa de las Naciones Unidas para el Desarrollo, el índice de pobreza multidimensional (IPM) identifica el nivel de carencia de los hogares en materia de salud, educación y niveles de vida. Accesible en http://hdr.undp.org/ en/node/2515 (18/05/20). 
superiores a la media nacional. Según el mismo organismo, el $55 \%$ de estos casos se concentraron en cuatro departamentos: Antioquia, Arauca, Cauca y Caquetá. Departamentos históricamente afectados por la violencia (ACNUDH, 2020).

Entre los municipios mayormente afectados por la comisión de crímenes contra la humanidad en el periodo 2016-2018 se encuentran Corinto, Puracé, Cajibio, Sucre, Mercaderes, Argelia, Piamonte (Cauca), Alto Baudó, Riosucio (Choco), El Rosario (Nariño), Córdoba, Puerto Escondido (Córdoba), Belén de los Andaquíes (Caquetá). Entre estos municipios están quienes superan el promedio de homicidios del país con un 23,07\% (CINEP, 2018). En contraste, según el Departamento Administrativo Nacional de Estadística (DANE), en relación con los índices de pobreza multidimensional para el 2018, Chocó obtuvo un 45,1\%, Córdoba 36,7\%, Nariño 33,5\%, Norte de Santander 31,5\%, Cauca $28,7 \%$, Caquetá $28,7 \%$, Putumayo $25,1 \%$, frente a la media nacional de $19,6 \%{ }^{10}$. Lo que representa un escenario de violencia estructural de acuerdo con los altos niveles de precarización social. En estos casos, a mayor presencia de violencia estructural, mayor probabilidad de violencia directa (Hueso, 2000).

Por su parte, en lo que tiene que ver con la política antidrogas de manera reactiva 0

10. El índice de pobreza multidimensional (IPM) mide las siguientes dimensiones: condiciones educativas de hogar, condiciones de la niñez y juventud, trabajo, salud, condiciones de vivienda y acceso a servicios públicos. Fuente: DANE, accesible en https://www.dane.gov.co/index.php/ estadisticas-por-tema/pobreza-y-condiciones-devida/pobreza-y-desigualdad/pobreza-monetariay-multidimensional-en-colombia-2018\#pobrezapor-departamentos-2018 (18/05/20). represiva, tampoco ha tenido los resultados prometidos, ya que, tras importantes esfuerzos en la aspersión o erradicación forzada de cultivos de uso ilícito, no se ha logrado eliminar por completo las hectáreas cultivadas, y la crisis en materia de derechos humanos continúa en estas comunidades. Las hectáreas de dicho cultivo se han visto en aumento, y han dejado a su paso miles de víctimas por cuenta de los grupos criminales al mando de estas economías ilegales. Las condiciones sociales y económicas presentes en estas zonas suponen un sustento de valor para el sostenimiento de las dinámicas del conflicto (Rios, 2016).

En estas circunstancias se requiere de instrumentos garantistas de los derechos humanos y políticas públicas eficaces que terminen con el sustento de recurso humano para las organizaciones criminales, y contribuyan con el bienestar social de la población. Así, el Estado debe hacer esfuerzos por combatir la violencia estructural, cultural y directa en un trabajo conjunto e interrelacional de sus instituciones. Ello de tal forma que, como plantea el Acuerdo de paz de 2016, lleguen las instituciones tanto en ámbitos como la justicia, políticas para el desmantelamiento de las organizaciones criminales, como en la generación de condiciones para los derechos humanos, en materia de inversión social y generación de oportunidades para la población.

Dado el histórico déficit de instituciones suficientemente contundentes para garantizar la producción de bienes públicos y la satisfacción de los derechos para todos los colombianos, es relevante un proyecto político de paz que contribuya con el desarrollo rural y la participación política. En palabras de Jaramillo Caro: "no hay posibilidad de que Colombia progrese 
de manera equitativa y sostenida si no resolvemos el problema del campo y clarificamos y garantizamos los derechos de propiedad de la tierra" (Jaramillo, 2015: 55).

De manera que el Acuerdo de paz de 2016 más que un instrumento para la transición de un conflicto vivo (con todas las deficiencias en implementación que han surgido hasta el momento), se ha proyectado como un acuerdo político que ofrece mecanismos significativos para la no repetición de crímenes contra la humanidad; sobre todo, en cuanto a la superación de la violencia estructural presente en los territorios más victimizados. En lo que sigue, se hace una aproximación al punto 1 del Acuerdo, como uno de los instrumentos relevantes en la superación del fenómeno de exclusión social en la población rural y consecuentemente, su contribución a la no repetición.

\section{El punto de reforma rural en el Acuerdo de paz: algunas proyecciones para la no repetición}

La demanda por la transformación de las condiciones estructurales en materia socioeconómica no aparece como una cuestión nueva en el debate social y político. Se reconoce que fenómenos como el desplazamiento, v. gr. en la región Pacífica, ha sido provocado tanto por la dinámica de la guerra interna -entiéndase entre guerrillas, y en general, entre las organizaciones criminales al mando de economías ilegales-, como por los procesos de modernización en el territorio, proyectos de extracción de los recursos naturales que muchas veces son ajenos a los intereses de las comunidades (Escobar, 2004). Como se ha reconocido, el monopolio de la tierra, del poder político y económico también han sido factores que han contribuido históricamente al escenario de violencia, con la cuestión agraria como uno de los elementos de la génesis del conflicto en el país (Matias, 2017).

Si bien es cierto que el ejercicio de la violencia sistemática ha afectado mayormente las zonas con presencia de grupos armados al margen de la ley y otros factores estratégicos (cultivos de uso ilícito, corredores, transporte, proyectos industriales, procesos de restitución de tierras, etc.), al mismo tiempo estas zonas se han caracterizado por grados considerables de precarización social. En este sentido, la consecución de una paz estable y duradera requiere de la voluntad para iniciar un nuevo orden social, económico y político con un enfoque territorial y participación ciudadana (Salas, 2016: 53). La apuesta por un escenario de paz en Colombia tiene que ver entonces con "respuestas concretas a las necesidades estructurales", con reformas que atiendan al sector excluido y permitan el desarrollo humano de las comunidades (Capera y Ñañez, 2017: 158).

En tanto que la comisión de crímenes contra la humanidad afecta a unos territorios más que a otros, resulta oportuno un enfoque territorial de derechos. El nuevo Acuerdo Final para la Terminación del Conflicto y la Construcción de una Paz Estable y Duradera ${ }^{11}$ pactado en 2016 entre el gobierno de Juan Manuel Santos (2010-2018) y la guerrilla de las Fuerzas

11. Acuerdo Final definitivo accesible en http://www.altocomisionadoparalapaz.gov.co/ procesos-y-conversaciones/Documentos\%20 compartidos/24-11-2016NuevoAcuerdoFinal. pdf (11/08/19). 
Armadas Revolucionarias de Colombia (FARC), además de ser un instrumento transicional para la desmovilización, representa un proyecto político que busca la transformación de las causas estructurales del conflicto; una de ellas, la situación de exclusión social de las zonas rurales. Si bien la apuesta del Acuerdo de paz de 2016 no representa una reconfiguración del sistema económico del país, plantea instrumentos que coadyuvan con la disminución de las brechas socioeconómicas entre la población rural y la urbana.

Con un enfoque en derechos humanos, además de ofrecer mecanismos de justicia y reparación a las víctimas, los puntos consignados en el Acuerdo, proyectan en su conjunto un esfuerzo integral para la no repetición de los crímenes contra la humanidad (Acuerdo final, 2016). A continuación, se realiza un esbozo sobre lo que proyecta el punto 1 del Acuerdo, titulado "Hacia un nuevo campo colombiano: Reforma Rural Integral". Ello sin olvidar que este tiene un carácter interdependiente que debe ir de la mano sobre todo con la cuestión de los cultivos de uso ilícito y la política de lucha en contra de las organizaciones criminales.

\subsection{La contribución a la no repetición desde un enfoque de derechos humanos}

Las garantías de no repetición como parte de los procesos de justicia transicional persiguen evitar el resurgimiento de los actores armados, legitimar el estado social de derecho, la democracia y los lazos de reconciliación (Duque y Torres, 2015). En el marco de garantías de no repetición se encuentran el desarme de los grupos armados al margen de la ley, la aplicación de la justicia para los criminales, los derechos a la verdad, y la reparación a las víctimas, entre otros (Romo, 2019). No obstante, en contextos como el colombiano, estas se corresponden con medidas a corto o mediano plazo que no garantizarían por sí solas, evitar un rearme de los grupos al margen de la ley, o la continuidad de la violencia sistemática. En este contexto, reducir la transición a estos elementos deja el camino abierto para la reaparición de las organizaciones criminales y consecuentemente la revictimización de la población.

Esto último puede entenderse en dos sentidos. Por un lado, en el caso de que la población vulnerable víctima de graves violaciones de derechos humanos regrese a contextos de exclusión social, y aquí la reparación "correctiva", o aquella con énfasis en el punitivismo no resulta la más eficaz. Como lo han expuesto autores como Uprimny (2009; 2016), en estos casos, poner a la víctima en la situación en la que se encontraba antes de su victimización -un principio de la justicia correctiva-, parece un acto de injusticia en sí, en términos de justicia distributiva. Ello en el entendido que estas víctimas padecían escenarios de exclusión social y discriminación antes del hecho criminal. De lo que deviene la necesidad de, no solo retribuir con castigo a los victimarios, sino también de transformar las condiciones de vulnerabilidad o precarización social en que se encontraba. Por el otro lado, transformar las condiciones de vulnerabilidad de las comunidades contribuiría con la diminución de la oportunidad que tienen las organizaciones criminales para reclutar el recurso humano.

En este punto cabe recordar que el debate de la justicia en los procesos transicionales, ha seguido una tendencia en 
separar la retribución y la restauración, la justicia y la paz. No obstante, los objetivos políticos como la paz y la reconciliación social suelen representar obligaciones prioritarias para los actores involucrados sobre todo en contextos de conflicto vivoque requieren que las medidas de un tipo de justicia y otro se articulen (Escudero, 2017). A este tipo de debates, las investigaciones de Uprimny (2009; 2016) y su propuesta sobre la "reparación transformadora" arroja luces en contextos como el colombiano. Donde se llama la atención sobre la necesidad de transformar las condiciones de exclusión social y vulnerabilidad en que se encuentra gran parte de las comunidades victimizadas.

Es por ello que los mecanismos que contribuyen a disminuir las causas estructurales de la violencia son fundamentales para la consecución y consolidación de una paz a largo plazo. Este tipo de medidas además de atender el derecho de las víctimas de manera integral con el impulso a la consecución de sus proyectos de vida, crean condiciones para garantizar los derechos humanos, económicos y sociales de las comunidades más afectadas. Se debe insistir en las garantías de corto y mediano plazo, pero de la mano de aquellas que a largo plazo, persigan tanto la prevención como la protección de los derechos humanos. Y, por supuesto, la transformación de la violencia estructural (Duque y Torres, 2015).

En el caso colombiano, los cambios estructurales deben estar guiados por cuestiones complejas como elementales: el desmantelamiento de las organizaciones criminales que no se han sometido a la justicia, la solución al narcotráfico, y la búsqueda de reformas para el respeto, protección y promoción de los derechos humanos, socioeconómicos, y culturales de la población. Es así que mecanismos como la Reforma Rural Integral, busca contribuir con la inclusión socioeconómica y cultural que permita las reconstrucción y fortalecimiento del tejido social. La no repetición, como punto que se compone de la integralidad del acuerdo, se caracteriza por su proyección en la protección de los derechos humanos, desde poner fin al conflicto, hasta garantizar los derechos que promete la constitución (Acuerdo final, 2016; Garay y Pérez, 2018).

De otro lado, frente al ejercicio de la violencia directa en los territorios, la estrategia del Estado ha girado en torno a soluciones e intervenciones de carácter militar, y parece dejar en un segundo plano las garantías para los derechos humanos de la población (Ardila, 2018: 185; ACNUDH, 2020). Si continúa la ausencia de una transformación estructural en los territorios, estos seguirán en contextos abonados para la criminalidad organizada y las comunidades más vulnerables seguirán expuestas a los embates de la violencia.

\subsection{La transformación del campo como contribución a la paz}

Como se ha reconocido, los programas en torno a una reforma rural deben tener como uno de sus objetivos la disminución de la brecha entre el campo y la ciudad, así como el mejoramiento de las condiciones de vida de las comunidades (Jaramillo, 2015). El punto 1 del Acuerdo persigue el establecimiento de un escenario de paz en las regiones con mayor nivel de precarización social y victimización. Propone un enfoque territorial y de derechos, ya que como es sabido, la vulneración de los mismos no ha sucedido con la 
misma intensidad en todos los territorios a lo largo de la historia del país (JaramiIlo, 2015). Se trata de un enfoque que apuesta por una transformación de los territorios más afectados por el conflicto, la violencia sistemática, y la vulneración de los derechos humanos.

El objetivo es contribuir con la disminución de la violencia directa, estructural, aunque también cultural por su proyección focalizada y con sentido territorial (Acuerdo final, 2016). Se busca la generación de oportunidades socioeconómicas que permitan transiciones y la inmersión del campo en el mercado nacional. Persigue entonces la inclusión de aquellos sectores rurales más afectados por las dinámicas del conflicto. Pues desde la apertura económica de los 90s, se ha visto la ausencia de políticas efectivas para incentivar la economía campesina de los pequeños productores (Tobón, 2018).

Pero para que estrategias como la erradicación de cultivos de uso ilícito sea exitosa, se requiere de un balance entre la erradicación y el desarrollo alternativo que a largo plazo garantice la sostenibilidad de la economía campesina ${ }^{12}$. En este escenario, la transformación del campo prioriza aspectos como el acceso a los servicios públicos, el mejoramiento de la infraestructura para el transporte y comunicación de los territorios, la inversión en gasto social y una mayor articulación de la política antidroga (Junguito, Perfetti, y Delgado, 2017).

12. Se debe tener en cuenta que particularmente el punto 4 sobre la sustitución de cultivos ilícitos va directamente relacionado con la reforma rural, y la eficaz ejecución interrelacional de estos puntos es obligatoria si se quiere la ejecución exitosa de estos (Tobón, 2018).
La propuesta del punto 1 trata entre otros aspectos, la transformación de la producción económica y los medios de vida mediante el desarrollo de proyectos económicos productivos. Donde además se gestione el territorio y la tierra, de manera que se generen condiciones de vida digna para la población. Desde tal sentido se busca financiación de proyectos productivos al tiempo que la gestión de políticas efectivas para la sustitución de cultivos de uso ilícito y la persecución de la criminalidad. Para lo cual se requiere priorizar la inversión pública en las zonas más afectadas por la violencia. Esto mediante aspectos como la mejora de infraestructura y servicios públicos, y el acceso a la tierra mediante la formalización, el catastro multipropósito, o el fondo de tierras, puntos tratados en la Reforma Rural (Junguito, Perfetti, y Delgado, 2017).

Esta Reforma Rural plantea la necesaria transformación de los territorios, en su mayoría habitados por comunidades étnicas y campesinas. Busca garantizar el acceso a la tierra, así como una democratización de la propiedad en beneficio de los que habitan el campo (Acuerdo final, 2016). En cuanto a los mecanismos operativos mediante los que se materializa la proyección del Acuerdo contienen desde componentes como el acceso y uso de la tierra, los Programas de Desarrollo con Enfoque Territorial PDET o los Planes Nacionales para la Reforma Rural Integral (Matias, 2017: 30). A grandes rasgos, estos elementos operativos pueden desglosarse como sigue:

1. Fondo de tierras: que tiene como fin la formalización masiva de la pequeña propiedad, los baldíos, la restitución, actualización del catastro rural, o la protección de las zonas de reserva campesina. 
2. Planes de Desarrollo con Enfoque Territorial PDET: planes de acción para la transformación de aquellas zonas rurales más afectadas por el conflicto en todas sus dimensiones. 3. Planes nacionales para la reforma rural integral ${ }^{13}$, que se refieren a la adecuación de infraestructura, electricidad y conectividad, servicios básicos, estímulos a la producción, subsidios, créditos en el campo, así como la generación de garantías para el derecho a alimentación, entre otros. Este último busca garantías para el acceso a la alimentación teniendo en cuenta que el campo cuenta con los más altos índices de pobreza multidimensional del país ${ }^{14}$ (Acuerdo final, 2016; Tobón, 2018).

Entre los objetivos del "Fondo de tierras para la Reforma Rural integral" se encuentra el acceso a la tierra para aquellos campesinos y campesinas que no poseen tierras o que poseen insuficiente, de las comunidades más golpeadas por la pobreza, el abandono institucional, y las dinámicas del conflicto; también la regularización de la propiedad y la promoción de la distribución equitativa de esta. En este sentido, se hace un reconocimiento a la economía campesina que al mismo tiempo se articula con otras formas de producción para la agricultura. En cuanto a los destinatarios de la adjudicación y los subsidios serán aquellos y aquellas

13. Estos tienen por objeto disminuir los niveles de pobreza y avanzar en la generación de bienestar para la población rural y una mayor integración entre el campo y la ciudad (Matias, 2017).

14. En lo que respecta a los puntos 1 y 4 del Acuerdo, de acuerdo con investigadores, estos no parecen representar una amenaza para el fisco del país, pues las inversiones previstas se encuentran dentro de las posibilidades de financiamiento por parte del gobierno nacional (Junguito, Perfetti y Delgado, 2017). con "vocación agraria" priorizando la población rural victimizada (Matias, 2017). Para un análisis de los mecanismos de la Reforma Rural véase, Matias, 2017; Junguito, Perfetti, y Delgado, 2017.

Programas como los PDET, por su parte, reconocen el carácter asimétrico del conflicto, porque priorizan territorios más afectados por la violencia. Ya que históricamente estos mismos han estado sometidos a niveles considerables de pobreza, o narcotráfico, y precariedad de las instituciones (Salas, 2016). Estos programas han propuesto como objetivo crear las condiciones para la transformación estructural de las zonas rurales y las regiones (Matias, 2017).

De otro lado, superar los altos índices de pobreza multidimensional no se reduce al aumento monetario de ingresos familiares (como subsidios), sino que además se precisa de la gestión de políticas públicas -v.gr., relacionamiento entre Ios PDET y los Planes Nacionales- para garantizar el acceso a los derechos más fundamentales (Matias, 2017). Estos son esenciales para el desarrollo humano y la disminución de las condiciones de precarización social. Tienen que ver con el imprescindible acceso a derechos como la educación, la salud, los servicios públicos, el acceso al mercado laboral en condiciones dignas, etc. Si bien esta reforma no implica una reestructuración del modelo económico (Matias, 2017), representa una contribución significativa para el progreso social de la población más vulnerable.

Pues bien, para hacer frente de manera integral a la agudización de la violencia sistemática, se requiere atender la problemática de la vulneración de derechos en territorio rural, que ha padecido el abandono y representa los mayores ín- 
dices de pobreza multidimensional en el país (Tobón, 2018). Como es sabido, estos territorios que han padecido mayores condiciones de precarización social, han sido al mismo tiempo los más afectados por el conflicto y la comisión de crímenes contra la humanidad. En consecuencia, la transformación de las condiciones estructurales de la violencia, necesitan entre otros, de la generación de oportunidades y garantías para el desenvolvimiento de la economía campesina, el desarrollo productivo y cultural con un enfoque territorial, así como de condiciones de vida digna en un sentido material (Acuerdo final, 2016).

Pero para que los mecanismos pactados tengan cabida, se necesita de la articulación de aquellas instancias encargadas de los proyectos de desarrollo rural y la gestión de la propiedad rural, al mismo tiempo que se procure una inversión pública eficaz en materia social. En este orden de ideas, el desarrollo de los proyectos deberá responder a las necesidades de la comunidad, generar instrumentos adecuados de participación y capacitación (Junguito, Perfetti, y Delgado, 2017). Ello teniendo en cuenta la necesaria articulación interinstitucional, así como la interdependencia de los puntos consignados.

La Reforma Rural Integral consignada en el acuerdo, proyecta así una contribución significativa para la disminución de los altos niveles de precarización y exclusión social de la población rural. Ello teniendo en cuenta que para su gestión se necesita del concurso de otros puntos que también se han establecido en el Acuerdo, véase, por ejemplo, la política para el desmantelamiento de las organizaciones criminales, o la solución a la cuestión de los cultivos ilícitos. Pero su consecución exitosa dependerá de la voluntad de los actores involucrados para llevarlos a cabo en su integralidad e interdependencia. Hoy, tal implementación ha devenido parcial y esto parece contribuir con la continuación y el círculo de la violencia (CINEP, CERAC, 2019).

\section{Conclusiones}

1. A lo largo del presente trabajo se ha desarrollado un análisis sobre la violencia estructural en el contexto colombiano, su relación con la comisión de crímenes contra la humanidad, y finalmente el enfoque de garantías de no repetición a partir del punto 1 del Acuerdo de paz de 2016. El objetivo ha sido comprender la importancia de atender la violencia estructural para la no repetición del círculo de violencia, cuestión demandada desde hace décadas y que ha quedado plasmada en la reforma rural del acuerdo. Para lo anterior se ha recurrido a una comprensión sociohistórica mediante la descripción de datos objetivos respecto del contexto de la violencia en Colombia, una revisión de los datos sobre crímenes contra la humanidad hacia líderes sociales, y una aproximación sobre la importancia de la reforma rural en la transformación de la violencia estructural.

2. Más allá de un punto de vista valorativo, una perspectiva sociológica que recurre a lo que ha demostrado la historia y el contexto del país, permite concluir que la violencia estructural ha contribuido significativamente al desarrollo y perpetuación del conflicto. Además, los datos descritos confirman que los crímenes contra la humanidad han afectado mayormente a las poblaciones más vulnerables. Así las cosas, las garantías para la no repetición de estos fenómenos de violencia deben contribuir con la transformación de las condi- 
ciones que han favorecido la marginación y exclusión social de estas comunidades.

3. Dicho de otro modo, si se parte de un estudio social e histórico, existe una relación entre las condiciones de precarización social y comisión de crímenes contra la humanidad en Colombia, o lo que es lo mismo, la relación entre violencia estructural y directa. Con lo cual, resulta significativo el punto de reforma rural -en su interrelación con otros-, en tanto que contribuye al mejoramiento de las estructuras sociales y económicas de los territorios más victimizados. La presencia institucional al tiempo que debe prestar atención en materia judicial o de persecución de la criminalidad organizada, debe hacer esfuerzos importantes para la generación de garantías sociales, económicas, y culturales en el territorio nacional. Sobre todo, respecto a los territorios priorizados por su vulnerabilidad en materia de derechos humanos.

4. Es preciso llamar la atención sobre la propuesta galtungiana de "paz por medios pacíficos". Pero en un sentido que va más allá de un optimismo humanista. Se debe propender por un proyecto político y económico que brinde herramientas de paz atendiendo a los factores sociales y materiales de la violencia. En el contexto colombiano, los medios pacíficos obedecerían, en el sentido aquí planteado, a la necesidad de atender la violencia estructural y consecuentemente invertir esfuerzos por la justicia social y la garantía de los derechos fundamentales.

5. La historia del conflicto colombiano confirma que la paz, o al menos, la posibilidad de esta, solo se podrá obtener por medios pacíficos. En esta línea y atendiendo al contexto, los cambios estructurales deben enfocarse hacia el desmante- lamiento de las estructuras criminales, así como al desenvolvimiento de las garantías para los derechos humanos. Esto último sobre todo en cuanto que la población más afectadas por la violencia directa sigue siendo aquella en condiciones de vulnerabilidad, aquellas zonas donde los niveles de pobreza multidimensional son significativos.

6. Las zonas que más han sufrido el flagelo de la violencia hacen un llamado a la integración socioeconómica, la protección de derechos humanos, y las garantías para lograrlo. Un esfuerzo en la estructuración de políticas públicas en esta materia, contribuiría a la restauración de los derechos de las víctimas, y también a la configuración de un terreno abonado para la no repetición de los crímenes contra la humanidad. El punto 1 del Acuerdo de paz de 2016 representa un esfuerzo significativo en este sentido. Pero para ser efectivo, se necesita de la voluntad política y de esfuerzos interinstitucionales que trabajen en la misma dirección.

\section{Bibliografía}

Acuerdo Final para la terminación del conflicto y la construcción de una paz estable y duradera. (12/11/2016). Accesible en https://www.cancilleria.gov.co/sites/default/files/Fotos2016/12.11_1.2016nuevo acuerdofinal. pdf 26/04/2020.

Alto Comisionado de las Naciones Unidas para los Derechos Humanos ACNUDH. (2019). Informe Anual del Alto Comisionado de las Naciones Unidas para los Derechos Humanos e informes de la Oficina del Alto Comisionado y del Secretario General. Accesible en: https://www.hchr.org.co/index. php/index.php/informes-y-documentos/ informes-anuales/9017-informe-del-altocomisionado-de-las-naciones-unidas-pa- 
ra-los-derechos-humanos-sobre-la-situacion-de-derechos-humanos-en-colombiadurante-el-ano-2018 (18/05/20).

Alto Comisionado de las Naciones Unidas para los Derechos Humanos ACNUDH. (2020). Informe Anual del Alto Comisionado de las Naciones Unidas para los Derechos Humanos e informes de la Oficina del Alto Comisionado y del Secretario General. Accesible en https://www.hchr. org.co/documentoseinformes/informes/ altocomisionado/informe-anual-2019-ES. pdf (18/05/20).

Ambos, K. (2013). "Crímenes de lesa humanidad y Corte Penal Internacional". Cuadernos de Derecho Penal, (9), pp. 95-140.

Alviar, H. (2010). "La redistribución de la propiedad en América Latina: ¿debemos perder la fe en el derecho?". Revista internacional de pensamiento político, I época, 5, pp. 91-121.

Ardila, E. (2018). Las fronteras judiciales en Colombia. Bogotá: Universidad Nacional de Colombia.

Ball, P., Rodríguez, C., y Rozo, V. (2018). Asesinatos de líderes sociales en Colombia en 2016-2017: una estimación del universo. Bogotá: Dejusticia. Accesible en https://cdn.dejusticia.org/wp-content/uploads/2018/09/ Asesinatos-de-1\%C3\%ADderes-sociales-en-Colombia-en-2016-2017-unaestimaci\%C3\%B3n-del-universo.pdf (11/11/2020).

Bello, M. (2003). El desplazamiento forzado en Colombia: acumulación de capital y exclusión social. Revista Aportes Andinos, 7, 8 p.

Bolívar, E. (2011). "Crímenes de lesa humanidad en el Derecho Penal Internacional". Principia Iuris, (15), pp. 159-175.

Bonett, M. (2001). "El conflicto colombiano y los retos del siglo XXI". Revista Desafíos, 4-5, pp. 165-191.
Calderón, P. (2009). "Teoría de conflictos de Johan Galtung". Revista de Paz y Conflictos, 2, pp. 60-81.

Cancio, M. (2010). Los delitos de terrorismo: estructura típica e injusto. Madrid: Editorial Reus.

Capera, J. y Ñañes, J. (2017). "Una perspectiva crítica sobre la violencia en Colombia en épocas del postconflicto". Utopía y Praxis Latinoamericana, 22, (78), pp. 153-162.

Centro de Investigación y Educación Popular (CINEP) et al. (2018). ¿Cuáles son los patrones? Asesinatos de líderes sociales en el Post Acuerdo. Recuperado de https:// www.cinep.org.co/publicaciones/es/producto/cuales-son-los-patrones-asesinatos-de-lideres-sociales-en-el-post-acuerdo/ (20/06/19).

Cerezo, A. (2010). El protagonismo de las víctimas en la elaboración de las leyes penales. Valencia: Tirant lo Blanch. Chinchón, J. (2012). "El concepto de impunidad a la luz del derecho internacional: una aproximación sistémica desde el derecho internacional penal y el derecho internacional de los derechos humanos". Revista Electrónica de Estudios Internacionales (REEI), (24), pp. 1-31.

Centro de Investigación y Educación Popular (CINEP), Centro de Recursos para el Análisis de Conflictos (CERAC). (2019). Sexto informe de verificación de la implementación del Acuerdo Final de Paz en Colombia para los Verificadores Internacionales. Bogotá, CINEP/ CERAC. Recuperado de https://www. verificacion.cerac.org.co/wp-content/uploads/2019/06/Sexto-informe-trimestralSecretar\%C3\%ADa-T\%C3\%A9cnica.pdf (21/08/19).

Duque C. y Torres, L. (2015). "Las garantías de no repetición como mecanismo permanente para la obtención de la paz". Universitas Estudiantes, 12, pp. 269-290. 
Echandía, C. (2008). "El fin de la invulnerabilidad de las FARC". Nueva Sociedad. Democracia y Política en América Latina, 217, pp. 4-13.

Egea, C. y Soledad, J. (2007). "Territorio, conflictos y migraciones en el contexto Colombiano". Cuadernos Geográficos, 40, pp. 185-194.

Escobar, A. (2004). "Desplazamientos, desarrollo y modernidad en el pacifico colombiano". En Restrepo E. y Rojas, A. (eds.). Conflicto e invisibilidad. Retos de los estudios de la gente negra en Colombia. Popayán: Editorial Universidad del Cauca, pp. 53-72.

Escudero, R. (2017). “Debatiendo sobre justicia transicional: memoria, perdón y castigo". En Larrañaga, P., Cerdio, J., Salazar, P. (coord.), Entre la libertad y la igualdad: Ensayos críticos sobre la obra de Rodolfo Vázquez. México: Universidad Nacional Autónoma de México. Instituto de Investigaciones Jurídicas, pp. 99-115.

Fariñas, M. (1998). "Los derechos humanos desde una perspectiva sociojuridica". Derechos y Libertades, pp. 355- 375.

Forer, A., López, C. (2010). Acerca de los crímenes de lesa humanidad y su aplicación en Colombia. Bogotá: Deutsche Gesellschaft für Technische Zusammenarbeit (GTZ).

Garay, C. y Pérez, A. (2018). "Los derechos humanos en Colombia: Acuerdo final de paz y su proyección en la política de seguridad y defensa", Revista Científica General José María Córdova, 16, (23), pp. 83-105.

García, R. (2019). "Contexto de una sociedad en Conflicto: Visión panorámica de la violencia estructural en Colombia". Justicia, 24 (36), pp. 1-19.

Gil, A. (2003). "Los crímenes contra la humanidad y el genocidio en el estatuto de la Corte Penal Internacional". Revista de Derecho Penal, (1), pp. 215-270.
Gil, A. (2014). "La expansión de los delitos de terrorismo en España a través de la reinterpretación jurisprudencial del concepto organización terrorista". Anuario de Derecho Penal y Ciencias Penales, 67, (1), pp.105-154.

Gil, A. (2016). "Crímenes contra la humanidad". EUNOMÍA. Revista en Cultura de la Legalidad, (10), pp. 202-215.

Gómez Isa, F. (dir.). (2008). Colombia en su laberinto: una mirada al conflicto. Madrid: Los Libros de la Catarata.

Hernández, E. (2012). Intervenir antes que anochezca. Mediaciones, intermediaciones, y diplomacias no violentas de base social en el conflicto armado colombiano. Bucaramanga: Universidad Autónoma de Bucaramanga.

Hueso, V. (2000). "Johan Galtung: La transformación de los conflictos por medios pacíficos". Revista Cuadernos de estrategia, 111, pp. 125-159.

Instituto de estudios para el desarrollo y la paz (Indepaz). (05/06/2020). Sigue la violencia de las brigadas militares de erradicación forzada. Accesible en http://www. indepaz.org.co/sigue-la-violencia-de-lasbrigadas-militares-de-erradicacion-forzada-indepaz-acpaz/ (11/11/2020).

Jaramillo, S. (2015). "La paz territorial". Revista Academia Libre, 12, pp. 53-60.

Jiménez-Bautista, F. (2012). "Conocer para comprender la violencia: origen, causas y realidad". Convergencia. Revista de ciencias sociales, 58, pp. 13-52.

Junguito, R.; Perfetti, J.; y Delgado, M. (2017). Acuerdo de paz: reforma rural, cultivos ilícitos, comunidades y costo fiscal. Colombia: Cuadernos Fedesarrollo. Landa, J. (2018). "Políticas de víctimas de la violencia política en España y el País Vasco: una reflexión a la luz del holocausto". Revista General de Derecho Penal, (29), pp. 1-50. 
Liñán, A. (2017). "La interpretación del término" población civil" como elemento del tipo en el crimen contra la humanidad". Revista Penal, (40), pp. 168-182.

Matias, S. (2017). "La Reforma Rural Integral, la terminación del conflicto armado y el problema agrario en Colombia". Revista Diálogos de Saberes, (46), pp.19-39.

Oficina del Alto Comisionado de las Naciones unidas para los derechos humanos (OACNUDH). (2019). Informe del Alto Comisionado de las Naciones Unidas para los Derechos Humanos sobre la situación de Derechos Humanos en Colombia durante el año 2018. Recuperado de https://www.hchr.org. co/index.php/informes-y-documentos/ informes-anuales/9017-informe-del-altocomisionado-de-las-naciones-unidas-para-los-derechos-humanos-sobre-la-situacion-de-derechos-humanos-en-colombiadurante-el-ano-2018 (28/08/19).

Oslender, UI. (2004). "Geografías de terror y desplazamiento forzado en el pacifico colombiano: conceptualizando el problema y buscando respuestas". En Restrepo E. y Rojas, A. (eds). Conflicto e invisibilidad. Retos de los estudios de la gente negra en Colombia. Popayán: Editorial Universidad del Cauca, pp. 35-52.

Pécaut, D. (1997). "Presente, pasado y futuro de la violencia". Análisis político, (30), pp. 3-36.

Rios, J. (2016). "Periferialización del conflicto armado colombiano (2002-2014)". Geopolítica(s). Revista de estudios sobre espacio y poder, 7 (2), pp. 251-275.

Romo, C. (2019). "Reflexiones para la aplicación de los mecanismos de justicia transicional para agentes del Estado en Colombia desde las exigencias del principio de garantías de no repetición". Diálogos de Derecho y Política, 24, pp. 64-82. Salas, L. (2016). "Conflicto armado y configuración territorial: elementos para la consolidación de la paz en Colombia". Bitácora 26, (2), pp. 45-57.

Tobón, G. (2018). "La reforma rural integral y los acuerdos de la habana en la solución a la problemática de las drogas". En Pastrana, E. y Gehring, H. (eds.) "La problemática del tráfico ilícito de drogas: impactos regionales y globales". Bogotá: Editorial Pontificia Universidad Javeriana, pp. 453-470.

Uprimny, R. (2009). Reparaciones transformadoras de violaciones masivas de derechos humanos entre justicia distributiva y justicia correctiva. Lección inaugural presentada como profesor visitante en la Universidad de Utrecht en la Cátedra Unesco de Educación para la Paz, los Derechos Humanos y la Democracia.

Uprimny, R. (2016). "Reparaciones transformadoras: un mecanismo para aliviar las tensiones entre justicia correctiva y justicia distributiva". En Garcia, M., Ceballos, M. (Eds.), Democracia, justicia y sociedad. Diez años de investigación en Dejusticia. Bogotá: Colección Dejusticia, pp. 741-754.

Valencia, G. y Cuartas, D. (2009). "Exclusión económica y violencia en Colombia, 1990-2008: una revisión de la literatura". Perfil de Coyuntura Económica, 14, pp. 113-134. 